\title{
Design, Analysis and Development of Improved Hollow Concrete Block Making Machine
}

\author{
Yemane Zemicheal \\ Mechanical Manufacturing and Automation \\ Tianjin University of Technology and Education, \\ Tianjin, China
}

\author{
Qi Houjun \\ Mechanical Manufacturing and Automation \\ Tianjin University of Technology and Education, \\ Tianjin, China
}

\begin{abstract}
This project report deals with the design and development of an improved hollow concrete block making machine. The machine lays a particular number of blocks over the platform and moves further to lay another set of blocks, so as to cover the casting platform, in a continuous casting process. This study attempts to solve the problems encountered on the existing hollow concrete block making machine by improving the design of basic components, increasing production rate and minimizing damages of uncured blocks during transfer. The design is based on data from literature review about Concrete in building construction, Concrete block machine developmental stage, how concrete block making machine works and manual calculation. To meet the objective based on the design principles several component mechanisms are improved and analytical design analysis, part drawings and assembling drawing are also done.
\end{abstract}

Keywords: Concrete block, block making machine, mould, compacting-vibrating system, tamper.

\section{INTRODUCTION}

The area of interest for this project is how to improve the concrete block making machine to be more productive so the cost of hollow blocks will be reduced. The improved concrete block making machine is smaller in size than the medium concrete block maker, more productive and cost effective than the conventional single block making machine. It produces about 2400 standard blocks per day, using three to six workers (two operator and three to four laborers'). The new machine has the advantage of being productive and movable to remote areas for construction.

Building of structures from individual units or masonry is one of the oldest forms of construction used by man. The utilitarian hollow concrete block has gained respect in the construction sites since its evolvement. Concrete masonry construction may cost slightly more, but builders and homeowners like its durability, strength and heat-retention qualities [1]. A concrete block is made of concrete that's been molded into the correct shape, and then allowed to harden and cure.

Block making machines have been improved on since Harmon $\mathrm{S}$. Palmer invented the first successful commercial machine used to make concrete block in 1900, what they are today [2]. The portable hollow concrete block making machine consists of a steel framework that holds the other components in place. There's a front hopper for pouring the wet cement mix, a frame that holds the metal concrete-block molds, a tamper for compacting the concrete in the molds and a mechanism for pushing the molded blocks out of the machine for drying and curing. The machine is equipped with the molds appropriate to the recommended hollow blocks to be made. The machine lays a particular number (four or five) of blocks over the platform and moves further manually to lay another set of blocks, so as to cover the casting platform, in a continuous casting process.

The Concrete Block Technology is a highly profitable business for micro and small scale building material producers and construction companies. In Ethiopia the market for concrete blocks is growing at a rapid rate especially in the areas where stones and burnt bricks are not easily available or are of poor quality.

The focus of this project was to modify the design, and development of a hollow concrete block making machine which is smaller in size than the medium automatic machine, movable, cheaper and yet, did not compromise efficiency and with more production rate and cost effective than the conventional single block making machine. This way more hollow concrete blocks will be made, which will enhance development of the construction.

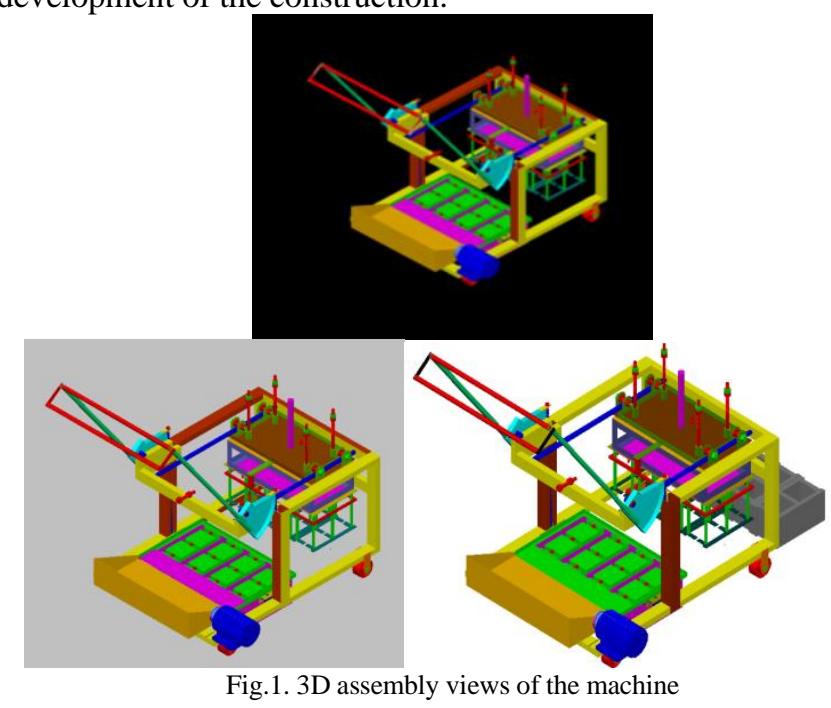

\section{Project objectives}

The objective of the project was to solve the problems encountered in the existing concrete block making machine by improving the design of main components leading to increased productivity. Generally the target was:

- To increase production rate of the hollow concrete block making machine

○ To minimize damages of uncured blocks during transfer

- To minimize power consumption and cost

- To make the machine easy and light for manipulating

○ To reduce foreign currency for importing the machine

O To encourage technology transfer in Ethiopia 


\section{II.DESIGN ANALYSIS}

\section{Description of the improved concrete block making machine}

Concrete Block Technology is based on the principle of densification of a lean concrete mix to make a regular shaped, uniform, high performance masonry unit. Concrete Block Technology can be easily adapted to suit special needs of users by modifying design parameters such as mix proportion, water/cement ratio and type of production system. It is an effective means of utilizing wastes generated by stone crushers, quarrying and stone processing units. The technology has high potential in areas where raw materials are easily available. The market for concrete blocks is growing at a rapid rate especially in the areas where stones and burnt bricks are not easily available or are of poor quality.

Table 4.2: Technical specifications of the machine

\begin{tabular}{|l|l|}
\hline \multicolumn{1}{|c|}{ Control } & \multicolumn{1}{c|}{ Manual } \\
\hline Usable area on Pallet & $500 \times 912 \mathrm{~mm}$ \\
\hline Maximum Block Height & $200 \mathrm{~mm}$. \\
\hline Frame Dimension (Width-Height-Length) & $1100 \times 1000 \times 1500 \mathrm{~mm}$ \\
\hline Total height of the machine & $1856 \mathrm{~mm}$ \\
\hline Frame Weight & $250 \mathrm{~kg}$ \\
\hline Material Bucket Capacity (hopper) & $40 \mathrm{lt}$. \\
\hline Vibrator motor & $2.2 \mathrm{~kW}(3 \mathrm{hp})$ \\
\hline
\end{tabular}

Table 4.3: Machine production capacity

Machine production Capacity

production Capacity per $\quad$ Hollow block (200x200x400)

shift (8 Hours)

\begin{tabular}{|l|l|}
\hline Hollow block $(200 \times 200 \times 400)$ & 1920 \\
\hline Hollow block $(150 \times 200 \times 400)$ & 2400 \\
\hline Hollow block $(100 \times 200 \times 400)$ & 3360 \\
\hline
\end{tabular}

- These technical values are based on standard specifications and may vary on customer's requirements, installation type and location.

Capacity value is theoretical value. It may vary according to aggregates structure, operator, weather and accommodation conditions.

i. The vibrations operations mode: - Eccentric weights, attached to the mould, vibrate the mold to provide the proper compaction of the concrete mix. The mold vibrates and produces cyclical sound levels. The vibration mode occurs only when the mix is being molded. As the block leaves the machine, the vibration ceases. Vibration lasts for about $6 \mathrm{sec}$ during each 30 -sec cycle. The timing of the cycling is highly dependent on the operating condition and production of the machine.

ii. Driving mechanism: - The machine uses an electrical motor for compaction purpose through pulley and belt mechanism.

iii. Appearance (aesthetic) of the machine: - It is good in appearance. Most of the parts are made to appear good for the operator and visitors, the handle arm, the frame, the trolley, the wheel and the motor stand are designed by taking the appearance of the machine in to consideration. Generally the machine is ergonomically good. (See Fig. 4.1)

\section{iv. Features of the machine:}

Salient features of the improved machine are given in the following:

○ A portable egg laying type machine.
- Four blocks of size $20 \times 20 \times 40 \mathrm{~cm}$ cast in one operation.

- Output of 200 to 300 blocks in an hour.

- Better compaction ensured through eccentric weight vibration for consolidation of concrete.

$\circ \quad$ One motor $3 \mathrm{hp}$ capacity and frequency $50 \mathrm{~Hz}$ used for power source.

- Suitable for casting common concrete blocks and different size hollow blocks.

- Operator's access right up to the moulds helps in easy placement of the mix in casting blocks and screeding of concrete mix in the moulds while casting hollow concrete blocks.

- Power required: $2.2 \mathrm{KW}$.

- It doesn't use pallets, just lays the blocks on a flat surfaces. After a set of blocks was produced and demoulded the machine moves forward to produce the next set of blocks and leaves the blocks on the floor for curing.

- It reduces time and energy consumption, because it uses almost the same time and energy which is used for producing one or two blocks at a time to produce four or five blocks.

- Amount of damaged blocks is minimized due to the avoidance of pallets and improvement of the sliding mechanisms.

- The overall size of the machine is minimized by removing unnecessary parts of the existing medium machine like supporter of the tamper, length of the frame, size of hopper and size of tamper trolley guide shaft for easy usage.

- The arm lever is part of the frame, the design and the shape of the arm is improved now it is simple for handling and manipulating.

- There is a stopper adjustment bolt for controlling the alignment between the mould and tamper during fitting or mating.

- Tamper release lever is in place for balanced and easy release of the tamper on both sides.

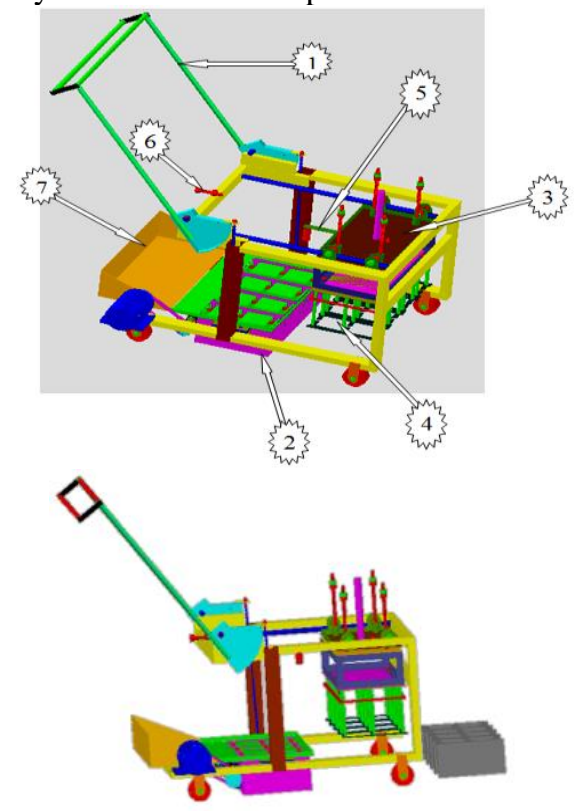

Figure 4.1 the newly designed hollow concrete block making machine 


\section{Design analysis of the critical parts}

The design was selected from an already made machine in the market with some modification in various parts and section to further enhance the productivity and functionality of the design. The concrete block making machine has a number of components but this topic focuses on the design of some of the components which are critical for proper functioning of the machine.

\section{i. The main shaft}

The shaft is fixed component of the machine which is normally circular in section that carries the tamper trolley assembly and sometimes including the blank mould. The main shaft of the machine is subjected to bending moment only.

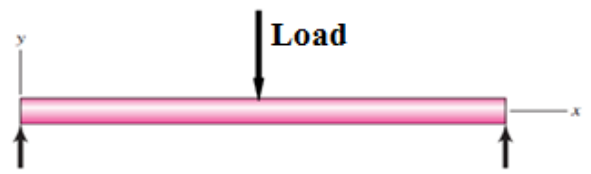

Figure 4.2 Main shaft free body diagram

The shaft is supported at both ends and the load is applied at the center. When the shaft is subjected to a bending moment only, then the maximum stress is given by the bending equation.

$$
\frac{M}{I}=\frac{\sigma_{b}}{y}
$$

Where $\mathrm{M}=$ Bending moment, $\mathrm{I}=$ Moment of inertia of crosssectional area of the shaft, $\sigma \mathrm{b}=$ Bending stress, and $\mathrm{y}=$ Distance from neutral axis to the outer-most fiber.

So by substituting some values

$$
M=\frac{\pi}{32} x \sigma_{b} x d^{3}
$$

To calculate the diameter of the shaft first lets calculate the weight applied.

Mass of the tamper trolley assembly is about $50 \mathrm{~kg}$ on each shaft and the length is $1.2 \mathrm{~m}$. The allowable bending stress is $75 \mathrm{Mpa}\left(75 \times 10^{6} \mathrm{~N} / \mathrm{m}^{2}\right)$.

$$
\mathrm{W}=\mathrm{m} \times \mathrm{g}=50 \times 9.81=490.5 \mathrm{~N}
$$

Maximum bending moment at the centre of the shaft,

$$
M=\frac{w \cdot L}{4}=\frac{490.5 \times 1.2}{4}=147.2 N-m
$$

Also we know that'

$$
M=\frac{\pi}{32} x \sigma_{b} x d^{3}
$$

And

$$
d^{3}=\frac{M \times 32}{\pi \times \sigma_{b}}=\frac{147.2 \times 32}{\pi \times 75 \times 10^{6}}, d=0.027 \mathrm{~m}
$$

$\therefore \mathrm{d}=27 \mathrm{~mm}$ take $30 \mathrm{~mm}$

Hence, the diameter of the main shaft to be produced is $30 \mathrm{~mm}$.

\section{ii. The vibrator-compactor system}

The main features of the vibrator-compactor system were the increased vibration of the die cavity or mould that was achieved through the attachment of an eccentric weight to the compactor drive shaft (cds). This caused the rotation of the cds to be irregular, and the mould to vibrate excessively as required for sand concrete compacting.

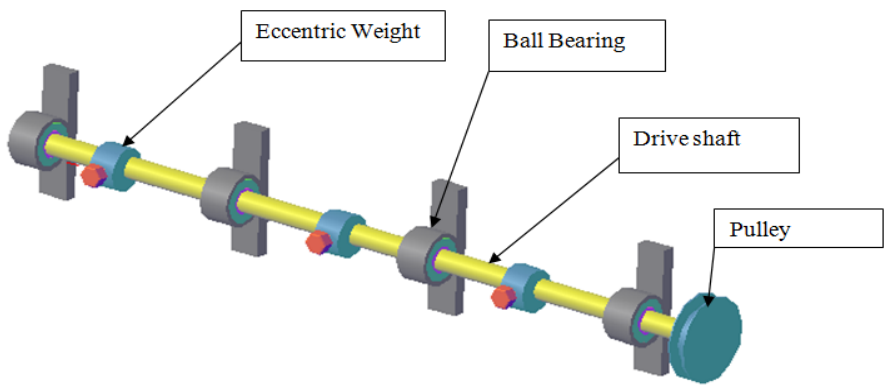

Figure 4.3: showing the compactor drive shaft and eccentric weight system

The eccentric weight is shown on figure 4.3 attached to cds. The length of cds inclusive of pulley is $30 \mathrm{~cm}$. the pulley is connected through a belt drive to a three horse power electric motor which drives the system. The eccentric weight and cds are made from mild steel material. The diameter and length of the eccentric weight (ew) are $6 \mathrm{~cm}$ and $11 \mathrm{~cm}$ respectively. The diameter and length of the cds are $3.0 \mathrm{~cm}$ and $30 \mathrm{~cm}$ respectively. The ew and cds are fastened with a bolt. The two flange ends of the cds are fitted into bearings housed in a disc. The ew and cds are encased by mild steel sheet.

The vibration caused by out-of-balance rotation when the ew and cds rotate is transmitted to the long face of the mould of the machine. The block mould was fabricated from a mild steel sheet of thickness $3 \mathrm{~mm}$. The block mould is rest in the ground when the machine is being operated. Mild steel frames are used to secure the block mould firmly in position in all its sides. The block mould is designed to move upwards along the inside of the frame to facilitate the ejection of moulded block. A lever or arm is attached for ejecting the moulded blocks.

\section{a. Theoretical Analysis of the compacting (vibrating) system}

The principle of the concrete (vibrator-compactor) block making machine is the eccentric weight. The eccentric weight, based on calculation for size, was fastened on the compactor drive shaft (cds). The cds was also based on design as carried out below. The attachment of eccentric to the cds caused an out-of-balance rotation that sets the long face of the mould to vibrate, a process which compacted the wet block mix.

a).

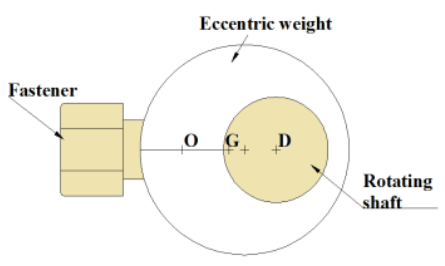

b).

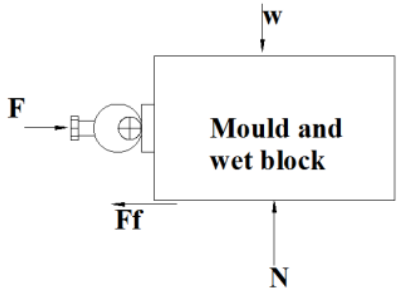

Figure 4.4: a) Eccentric weight Attached to Compactor Drive Shaft and b) free body diagram of the system. 
The rotation of the shaft gave rise to centrifugal force, Fc, which acted radially outwards in a direction that is perpendicular to the axis of rotation of the compactor drive shaft, and through the centre of gravity, G.

Centripetal force is defined as a force that pulls a rotating or spinning object toward a center or axis [Encarta Dictionary]. According to Newton's third law of motion, every action must have a reaction. Therefore the reaction to centripetal force is called centrifugal force [6]. Centrifugal force is defined as the force that pulls an object away from the center point. However, since an object would only move tangentially away from the center on a linear path (force of velocity), and not continue around a center point, centrifugal force is actually not a force at all, but rather a concept that represents an effect. Some definitions even describe this as an imagined force. This is interesting to keep in mind when applying these ideas to vibrator-compactor system.

The centripetal force Fp, was equal to Fc but in the opposite direction towards the centre of rotation. There were four forces that were relevant in design of the vibrator-compactor system of the machine. They were the centrifugal force, $F_{c}$, the spring force, Fs, the force due to flexural rigidity, Ff, and the force due to the wet block mix, $F_{w b}$. The point $O$ (fig 4.4) was the geometric centre of the eccentric weight, $G$ was the centre of gravity of the cds and eccentric, me was mass of eccentric, $\mathrm{m}_{\mathrm{wb}}$ was mass of wet block mix, $\mathrm{y}_{1}$ was the length OD and was the additional deflection of the centre of the cds when it rotated at $\omega \mathrm{rad} / \mathrm{s}$, e was the length OG which was the initial distance of the centre of the eccentric from the combined centre of gravity of the cds and eccentric weight, $\mathrm{K}$ was the stiffness of cds, it is the ability of a material to resist deformation under stress. The modulus of elasticity is the measure of stiffness, EI was the flexural rigidity of the cds, E was the young's Modulus of the cds, I was the moment of inertia of the cds, Ls was taken as the length of the compactor drive shaft, Le as the length of the eccentric, $y_{2}$ was half the diameter of cds and line of action of the flexural rigidity and $\mathrm{g}$ as acceleration due to gravity.

The design required that the following quantities were known:
1. The forces activated in the system,
2. The mass of the cds and eccentric,
3. Force due to wet block $F_{w b}$,
4. Operating stress,
5. The diameter of the cds and eccentric,
6. The length of the cds and eccentric,
7. The lengths of $y$ and $e$ for the cds and eccentric.

b. Forces Activated in The compacting-vibrating system: These were found to be four in number,

(i) The centrifugal force due to the cds and the eccentric weight $\mathrm{Fc}$, which was stated in,

$$
\mathrm{F}_{\mathrm{c}}=\mathrm{m}_{\mathrm{e}} \omega^{2}\left(\mathrm{y}_{1}+\mathrm{e}\right)
$$

Where $\mathrm{m}_{\mathrm{e}}, \omega, \mathrm{y}_{1}$, and $\mathrm{e}$ are as stated above, $\mathrm{F}_{\mathrm{c}}$ acted away from the axial centre of the cds perpendicularly.

(ii) The spring force in the cds, Fs. it is given in ;

$\mathrm{F}_{\mathrm{s}}=\mathrm{ky}_{1} \quad \ldots \ldots \ldots$

Where $\mathrm{k}$ is the stiffness of cds.

(iii) The force due to the flexural rigidity of the cds, Ff. The equation for $\mathrm{Ff}$ is given as;

$$
F_{f}=\frac{K E I}{L_{s}{ }^{3}} \mathrm{y}_{2}
$$

Where $\mathrm{K}, \mathrm{E}, \mathrm{I}, \mathrm{L}$ and $\mathrm{y}_{2}$ are stated above.

(iv) The force due to the wet block mix, $F_{w b}$ and is given as;

$\mathrm{F}_{\mathrm{wb}}=\mathrm{m}_{\mathrm{wb}} \mathrm{xg} \ldots \ldots$

Where $\mathrm{m}_{\mathrm{wb}}$ and $\mathrm{g}$ are as defined above.

\section{c. The Mass of the Eccentric and cds;}

A $2.2 \mathrm{hp}$ three phase electric motor, which ran at $2840 \mathrm{rpm}$, was selected because it was available in the market. The speed of rotation of motor

$\omega=\frac{2 \pi 2840}{60}=297.4 \mathrm{rad} / \mathrm{sec}$

For vibration to be propagated continuously, the eccentricity ratio was maintained at $\mathrm{y}_{1} / \mathrm{e}>1$.

Also for the equilibrium, equation (1) and (2) were equal, i.e. $\mathrm{Ky}_{1}=\mathrm{m}_{\mathrm{e}} \omega^{2}\left(\mathrm{y}_{1}+\mathrm{e}\right) \quad \ldots-\ldots$

with $\mathrm{y}_{1}: \mathrm{e}=1: 1$,

$\mathrm{ky}_{1}=\mathrm{m}_{\mathrm{e}} \omega^{2}(1+1) \mathrm{y}_{1}$

$m_{e}=\frac{31000 \times 1}{297.4^{2} \times 2}=0.18 \mathrm{~kg}$

mild steel)

For $\frac{y_{1}}{e}=1 \frac{1}{2}, 2,2 \frac{1}{2}, 3$ and 4, calculated values of $m_{e}$ were $0.21,0.23,0.25,0.26$, and $0.28 \mathrm{~kg}$ respectively. Selection of $\mathrm{m}_{\mathrm{e}}=0.21,0.23$, and did not give as adequate a vibration response as did 0.26 . The bolt and nut which fastened the eccentric weight to the cds, increased $\mathrm{m}_{\mathrm{e}}$ to $0.50 \mathrm{~kg}$. The weight of the cds was a half that of eccentric by design, i.e. $m_{s}=\frac{1}{2} m_{e}=0.25 \mathrm{~kg}$

\section{d. Force Due to Wet Block (Fwb);}

This is given in, $F_{w b}=m_{w b} \times g \times \mu \quad$ (one dry 20x20x40cm block $=17 \mathrm{~kg}$ ), and the mass of four blocks will be distribute to the three eccentric weights in the cds.

$\mathrm{F}_{\mathrm{wb}}=101.6 \mathrm{~kg} \times 9.81 \times 0.4=398.6 \mathrm{~N}$ - with wet block being $20 \%$ heavier than dry block including weight of the mould $20 \mathrm{~kg}$ and $\mu=0.3-0.7$ select 0.4 .

\section{e. Operating Stress, $\sigma$;}

This is given in,

$$
\sigma=\frac{\text { Force of } \text { wet block }}{\text { Area of } \text { cds }}=\frac{398.6}{\pi(0.0125)^{2}}=813.5 \mathrm{kN} / \mathrm{m}^{2}
$$

\section{f. Diameter of Eccentric Weight and Compactor Drive Shaft (cds);}

The cds carried the weight of the wet block mix in bending under minimum tension with a combined shock and fatigue factor of 1.5 [4]. The drive torque, $\mathrm{T}$, was given in,

$T=\frac{P x 60}{2 \pi N}=\frac{2200 \times 60}{2 \pi \times 2840}$ for the $3 \mathrm{hp}$ motor which ran at

2840rpm and $\mathrm{P}=2.2 \mathrm{kw}$

$\therefore \mathrm{T}=7.4 \mathrm{Nm}$

The cds in bending was expressed as;

$\left(\mathrm{m}_{\mathrm{wb}} \times \mathrm{g} \mathrm{x} \mu \mathrm{x} \mathrm{r}_{\mathrm{s}}\right) \times 1.5=7.4 \mathrm{Nm}$

Where $\mathrm{r}_{\mathrm{s}}=$ radius of cds $\therefore$

$$
r_{s}=\frac{7.4}{(101.6) \times 9.81 \times 0.4 \times 1.5}=0.01237 \mathrm{~m}
$$

Chose $r_{\mathrm{s}}=0.0125 \mathrm{~m}$

For the eccentric weight, the radius was calculated as, 
$r_{e}=\frac{2 \times 7.4}{(101.6) \times 9.81 \times 0.4 \times 1.5}=0.0247 \mathrm{~m}$

Where $r_{e}$ is the radius of eccentric weight, given wet block mix as $20 \%$ heavier than dry brick of weight $81.6 \mathrm{~kg}$.

Chose $r_{e}=0.025 \mathrm{~m}$.

Hence, the design diameters of the cds and eccentric weight were $2.5 \mathrm{~cm}$ and $5.0 \mathrm{~cm}$ respectively.

g. Length of compactor Drive Shaft and Eccentric Weight Density of cds (made from mild steel);

$\rho_{s}=\frac{m_{s}}{v_{s}}=7850 \mathrm{~kg} / \mathrm{m}^{3}$

i.e $\rho_{s}=\frac{m_{s}}{A_{s} \times L_{s}}=7850$

where $A_{s}$ and $L_{s}$ were area and length of the cds respectively. $L_{s}=\frac{m_{s}}{\rho_{s} v_{s}}=\frac{0.25 \times 20 \times 10^{4}}{7850 \times \pi \times 2.5^{2}}=32.4 \mathrm{~cm}$

Length of eccentric weight $\mathrm{L}_{\mathrm{e}}$ was;

$L_{e}=\frac{m_{e}}{\rho_{s} A_{s}}=\frac{0.5 \times 20 \times 10^{4}}{7850 \times \pi \times 5^{2}}=16.2 \mathrm{~cm}$

\section{h. Length of y1 and e for cds and Eccentric}

For $\mathrm{y}_{1}: \mathrm{e}=3: 1$, where $\mathrm{y}_{1}$ was the length of the centre of rotation from the geometric centre OD and e was the length of the centre of gravity from the geometric centre OG (Figure 4.4), the maximum force is assumed to also be three times the wet block force as

$\therefore \widehat{F}=\overline{F_{w b}} \times 3$

Where $\hat{F}_{\text {}}$ maximum allowed force.

$$
\begin{aligned}
\therefore \hat{F} & =398.6 \times 3=1195.8 \mathrm{~N} \\
\hat{F} & =\mathrm{m}_{\mathrm{e}} \omega^{2}\left(\mathrm{y}_{1}+\mathrm{e}\right) \\
\mathrm{y}_{1}+\mathrm{e} & =\frac{1195.8}{0.5 \times 297.4^{2}} \Rightarrow\left(1+\frac{1}{3}\right) \mathrm{y}_{1}=2.7 \Rightarrow \mathrm{y}_{1}=2.02 \mathrm{~cm} \\
& \frac{2.02}{3}=0.67 \mathrm{~cm} \\
\mathrm{e} & =2.02 \mathrm{~cm} \text { and } \mathrm{e}=0.67 \mathrm{~cm}
\end{aligned}
$$

\section{iii. Bearing}

Bearings are considered to be the most critical components of machinery. From specification description and standard table for $25 \mathrm{~mm}$ diameter shaft bearing the basic load rating: - For the medium series ball bearings the design life is 5 years and design life hour is $10000 \mathrm{hr}$ (from table), the required value $\left(\frac{C}{P}\right)=10.5$ $\operatorname{radial} \operatorname{load}(\mathrm{N})$ and $\mathrm{F}_{\mathrm{r}}=$ radial load.

Then the required basic load rating $(\mathrm{C})=(\mathrm{C} / \mathrm{P}) \mathrm{P}$, for radial load only $\Rightarrow \mathrm{P}=\mathrm{F}_{\mathrm{r}}$, so $\mathrm{P}=1195.8 \mathrm{~N}$ from the compactor vibrator design part

$$
\therefore \text { Basic load rating }
$$

$$
C=\left(\frac{C}{P}\right) P=10.5 \times 1195.8 \mathrm{~N}=12555.9 \mathrm{~N}=12.5 \mathrm{kN}
$$

And the bearing number 305 having $\mathrm{C}=12.5 \mathrm{kN}$, is selected. That means the bearing is of medium series whose bore is 05 $\times 5$, i.e., $25 \mathrm{~mm}$, outside diameter $=62 \mathrm{~mm}$ and width $=17 \mathrm{~mm}$.

iv. Pulley
The dimensions for the standard V-grooved pulley (Refer Fig. 4.11) are shown in standard Tables, from which we find that for ' $A$ ' type belt $\mathrm{w}=11 \mathrm{~mm}, \mathrm{~d}=12 \mathrm{~mm}, \mathrm{a}=3.3 \mathrm{~mm}, \mathrm{c}=8.7 \mathrm{~mm}, \mathrm{f}=10 \mathrm{~mm}$, and $\mathrm{e}=15 \mathrm{~mm} .(2 \beta=34)$

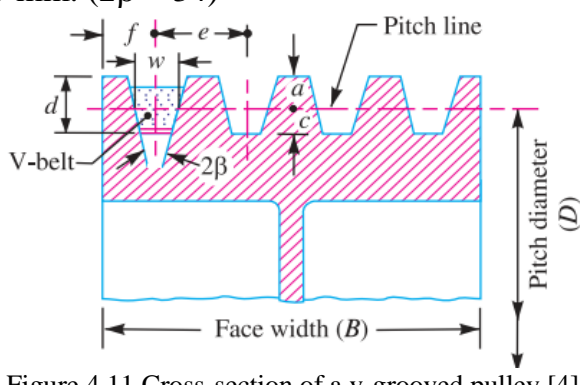

Figure 4.11 Cross-section of a v-grooved pulley [4]

We know that face width of the pulley,

$B=(n-1) e+2 f=(2-1) 15+2 \times 10=35 \mathrm{~mm}$

v. Key

A key is a piece of mild steel inserted between the shaft and hub or boss of the pulley to connect these together in order to prevent relative motion between them. It is always inserted parallel to the axis of the shaft. Keys are used as temporary fastenings and are subjected to considerable crushing and shearing stresses.

The rectangular key is designed as follows:

From data books we find that:

width of the key $(w)=d / 4=30 / 4=7.5 \mathrm{~mm}$

Thickness of the key $(\mathrm{t})=2 \mathrm{w} / 3=\mathrm{d} / 6=30 / 6=5 \mathrm{~mm}$

In order to find the length of the key to transmit full power of the shaft, the shearing strength of the key is equal to the torsional shear strength of the shaft.

We know that the shearing strength of key,

$T=l \times w \times \tau \times \frac{\mathrm{d}}{2}$ and torsional shear strength of the shaft,

$T=\frac{\pi}{16} \mathrm{x} \tau_{1} \mathrm{xd}^{3}$

From the above equations we have,

$l \times \mathrm{w} \times \tau \times \frac{\mathrm{d}}{2}=\frac{\pi}{16} \times \tau_{1} \mathrm{xd}^{3}$

$l=\frac{\pi}{8} x \frac{\tau_{1} d^{2}}{w \times \tau}=\frac{\pi d}{2} x \frac{\tau_{1}}{\tau}=1.571 d x \frac{\tau_{1}}{\tau}$

----------- (Taking $\mathrm{w}=\mathrm{d} / 4)$

When the key material is same as that of the shaft, then $\tau=$ $\tau 1$

Then

$$
l=1.571 \mathrm{~d}=1.571 \times 30=47.13 \mathrm{~mm}=47 \mathrm{~mm}
$$

\section{CONCLUSION AND RECOMMENDATIONS}

The concrete block making machine is designed to produce four blocks at a time. It can also produce five blocks at a time by changing the mould and tamper assembly to the desired dimension. On the design in addition to the compactingvibrating system the weight of the machine is considered in relation to the personnel for moving the machine and ease of operation. The improved machine is suitable for the production of hollow blocks inside of halls and outside, just a leveled concrete floor is needed. It can be fabricated locally and from the accessible materials. The cost of the machine is 
comparatively low and can be affordable by both poor and rich; it requires little or no frequent maintenance. Generally, the results of this study are optimistic, providing a good starting point for further exploration on the same area. The Technology can be easily adapted to suit special needs of users by modifying design parameters such as mix proportion, water/cement ratio and type of production system.

In conclusion the following are recommended to be investigated further:

$>$ Mechanism to minimize the initial cost for platform concrete

> Designing other additional accessories and systems that make the current design to become more ease for operation.

Making the machine more ease and simple in relation to ergonomic design.

The researchers sincerely wishes that improvement in the concrete block making machine technology in Ethiopia that stepped here to continue and get improved by faculties, students and other concerned bodies. We are also interested to continue the improvement practice in the future to make it more advanced in compacting, power consumption and production rate.

\section{ACKNOWLEDGMENT}

The authors would like to acknowledge that some students and instructors at Adama science and Technology University were involved in developing the Design, Analysis and Development of Improved Hollow Concrete Block Making Machine. The author wish to thank Dr. Mengist Hailemariam for his invaluable support to this work.

\section{REFERENCES}

[1] Don Vandervort, (2014). Concrete Building Blocks, HOME TIPS. https://www.hometips.com/buying-guides/concrete-cinderblocks-construction.html

[2] Hall, J. P. (2009). The early developmental history of concrete block in America.

[3] Juvinall, R. C. (2003). Fundamentals of machine component design, fourth reversed edition.
[4] Khurmi, R. S., \& Gupta, J. K. (2005). A text book of Machine Design. Eurasia Publishing house (PVT.) Ltd. First multi color edition, 576-586.

[5] Kl. Narayana, (2006). Machine Drawing, third edition, new age international, New Delhi.

[6] Laura J. LaLiberte, (2009). Centripetal \& centrifugal forces, Florida Atlantic University, Florida.

[7] VDOKUMENTS, Chapter 2 manufacturing: History, manufacturing methods and quality control of concrete blocks. https://vdocuments.mx/parta-ch02.html

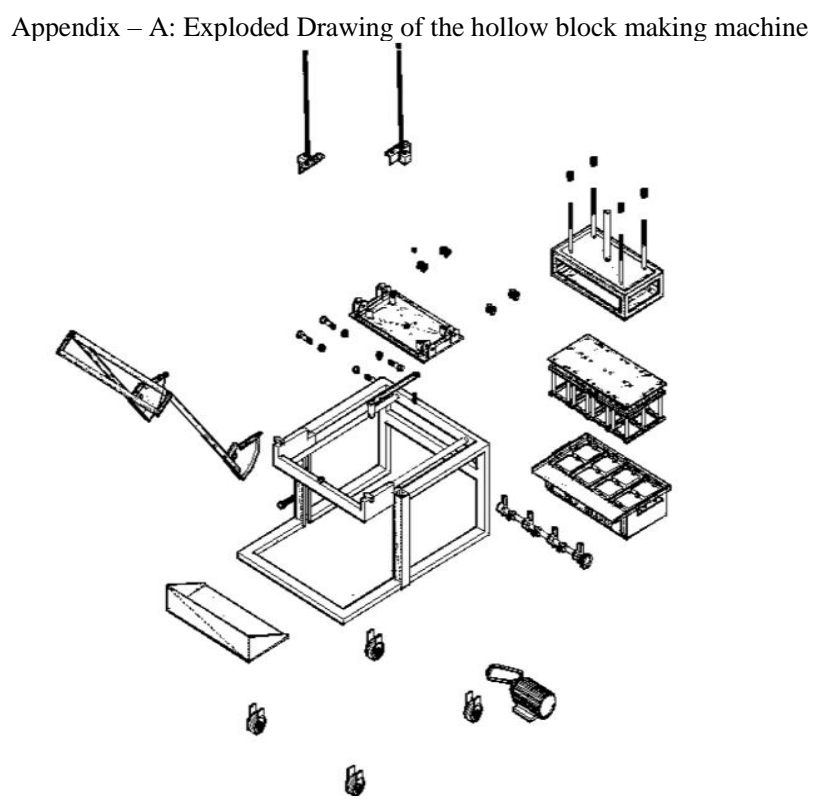

